\title{
A PROBLEM INVOLVING A NONLOCAL OPERATOR
}

\author{
RATAN Kr. Giri, D. ChOUdhuri And Amita Soni
}

Abstract. The aim of this paper is to deal with the elliptic pdes involving a nonlinear integrodifferential operator which are possibly degenerate and covers the case of fractional $p$-Laplacian operator. We prove the existence of a solution in the weak sense to the problem

$$
\begin{aligned}
-\mathscr{L}_{\Phi} u & =\lambda|u|^{q-2} u \text { in } \Omega, \\
u & =0 \text { in } \mathbb{R}^{N} \backslash \Omega,
\end{aligned}
$$

if and only if a weak solution to

$$
\begin{aligned}
-\mathscr{L}_{\Phi} u & =\lambda|u|^{q-2} u+f, f(\neq 0) \in L^{p^{\prime}}(\Omega), \\
u & =0 \text { in } \mathbb{R}^{N} \backslash \Omega,
\end{aligned}
$$

( $p^{\prime}$ being the conjugate of $p$ ), exists for $q \in\left(p, p_{s}^{*}\right)$ under certain condition on $\lambda$, where $-\mathscr{L}_{\Phi}$ is a general nonlocal integro-differential operator of order $s \in(0,1)$ and $p_{s}^{*}$ is the fractional Sobolev conjugate of $p$. We further prove a necessary condition for the existence of a weak solution to the problem

$$
\begin{aligned}
-\mathscr{L}_{\Phi} u & =\lambda|u|^{q-2} u+\mu \text { in } \Omega, \\
u & =0 \text { in } \mathbb{R}^{N} \backslash \Omega,
\end{aligned}
$$

where $\mu$ is a measure.

Mathematics subject classification (2010): Primary 35J35, secondary 35J60. space etc.

Keywords and phrases: Nonlocal operators, fractional $p$-Laplacian, elliptic PDE, fractional Sobolev

\section{REFERENCES}

[1] B. Abdellaoui, A. Attar And R. Bentifour, On the Fractional p-Laplacian equations with weight and general datum, Adv. Nonlinear Anal., (2016) (arXiv:1601.00606 [math.AP]).

[2] A. BAhri AND H. BerestycKI, A perturbation method in critical point theory and applications, Trans. Amer. Math. Soc. 267, 1 (1981), 1-32.

[3] A. BAhri And P. L. Lions, Morse index of some min-max critical points, I. Application to multiplicity results, Commun. pure appl. math. 41, 8 (1988), 1027-1037.

[4] B. Barrios, E. Colorado, R. Servadei and F. Soria, A critical fractional equation with concave-convex power nonlinearities, Ann. Inst. H. Poincaŕe Anal. Non Linéaire 32 (2015), 875-900.

[5] C. Brändle, E. Colorado, A. Pablo And U. Sánchez, A concave-convex elliptic problem involving the fractional Laplacian, Proc. Roy. Soc. Edinburgh. Sect. A. 143 (2013), 39-71.

[6] C. Bucur And E. VAldinoci, Nonlocal diffusion and applications, Springer international publishing, Switzerland, 20, 2016.

[7] X. CABRÉ AND J. TAN, Positive solutions of nonlinear problems involving the square root of the Laplacian, Adv. Math. 224 (2010), 2052-2093.

[8] L. Caffarelli, Non-local diffusions, drifts and games, Nonlinear Partial Differential Equations, Abel Symposia 7 (2012), 37-52. 
[9] L. CAfFarelli And L. Silvestre, An Extension problem related to the fractional Laplacian, Commun. Partial Differential Equations 32 (2007), 1245-1260.

[10] D. Choudhuri AND A. Soni, Existence of multiple solutions to a partial differential equation involving the fractional $p$-Laplacian, J. Analysis 23 (2015), 33-46.

[11] E. Di NezZA, G. PALAtucCi, E. VAldinOci, Hitchhiker's guide to the fractional Sobolev spaces, 2011, Equipe MIPA.

[12] L. C. Evans, Partial Differential Equations, Amer. Math. Soc., 2009.

[13] G. Franzina and G. Palatucci, Fractional p-eigenvalues, Riv. Mat. Univ. Parma (N. S.) 2, 5 (2014), 315-328.

[14] T. Gallouët, Jean-Michel Morel, Resolution of a semilinear equation in $L^{1}$, Proc. of the Royal Soc. of Edinburgh 96A (1984), 275-288.

[15] J. P. Garcia Azorero And I. Peral Alonso, Existence and uniqueness for the p-Laplacian: nonlinear eigenvalues, Commun. in partial differential equations 12, 12 (1987), 1389-1430.

[16] R. KR. GiRI AND D. ChoudhuRI, A problem involving the p-Laplacian operator, Differ. Equ. Appl. 9, 2 (2017), 171-181.

[17] S. Goyal AND K. SREenAdH, Nehari manifold for non-local elliptic operator with concave-convex nonlinearities and sign-changing weight functions, Proc. Indian Acad. Sci. (Math. Sci.) 125, 4 (2015), $545-558$.

[18] A. IAnnizzotto AND M. SQuassina, Weyl-type laws for fractional p-eigenvalue problems, Asymptotic Analysis 88, 4 (2014), 233-245.

[19] S. KeSAVAn, Topics in Functional Analysis and applications, New age international pvt. 1td., 2003.

[20] T. Kussi, G. Mingione And Y. Sire, Nonlocal equations with measure data, Commun. Math. Phys. 337, 3 (2015), 1317-1368.

[21] E. Lindgren And P. Lindqvist, Fractional eigenvalues, Calc. Var. Partial Differential Equations 49, 1-2 (2014), 795-826.

[22] G. Molica BISCI AND R. SERVADEI, Lower semicontinuity of functionals of fractional type and applications to nonlocal equations with critical Sobolev exponent, Adv. Differential Equations 20 (2015), 635-660.

[23] G. Molica Bisci AND R. SERVAdeI, A Brezis-Nirenberg splitting approach for nonlocal fractional equations, Nonlinear Anal. 119 (2015), 341-353.

[24] S. Mosconi, K. Perera, M. Squassina And Y. Yang, The Brezis-Nirenberg problem for the fractional p-Laplacian, (arXiv:1508.00700v1 [math.AP]).

[25] T. MuKheRJEe AND K. SREenAdh, On Dirichlet problem for fractional p-Laplacian with singular nonlinearity, (arXiv:1602.00872v2 [math.AP]).

[26] P. Piersanti AND P. PUCCI, Existence theorems for fractional p-Laplacian problems, Anal. Appl. 15, 5 (2017), 607-640.

[27] P. PUCCI, S. SALDI, Asymptotic stability for nonlinear damped Kirchhoff systems involving the fractional p-Laplacian operator, J. Differetial Equations 263, 5 (2017), 2375-2418.

[28] R. Servadei And E. VAldinoci, Mountain pass solutions for non-local elliptic operators, J. Math. Anal. Appl. 389, 2 (2012), 887-898.

[29] R. SERVADEI AND E. VAlDINOCI, Variational methods for non-local operators of elliptic type, Discrete Contin Dyn. Syst. 33, 5 (2013), 2105-2137.

[30] R. SERVAdei And E. VAldinoci, The Brezis-Nirenberg result for the fractional Laplacian, Trans. Amer. Math. Soc. 367, 1 (2015), 67-102.

[31] X. SU AND Y. WEI, Multiplicity of solutions for non-local elliptic equations driven by the fractional Laplacian, Calc. Var. Partial Differential Equations 52, 1-2 (2015), 95-124.

[32] G. TaRantello, On nonhomogeneous elliptic equations involving critical Sobolev exponent, Ann. Inst. H. Poincaŕe 9C, 3 (1992), 281-304.

[33] L. VÁzQUEZ, Nonlinear diffusion with fractional Laplacian operators, in Nonlinear Partial Differential Equations: the Abel Symposium 2010, Holden, Helge and Karlsen, Kenneth H. (eds.), Springer, 2012, 271-298.

[34] X. YU, The Nehari manifold for elliptic equation involving the square root of the Laplacian, J. Differential Equations 252, 2 (2012), 1283-1308. 